\title{
BOUNDARY BEHAVIOR OF BLASCHKE PRODUCTS IN THE UNIT CIRCLE
}

\author{
J. R. KINNEY ${ }^{1}$
}

Let $\left\{a_{j}\right\}_{j=1,2,} \ldots$ be a sequence with $0<\left|a_{j}\right|<1$ and $\sum_{j \geq 1}\left(1-\left|a_{j}\right|\right)$ $<\infty$. The Blaschke product defined by

$$
B(z)=\prod_{j \geq 1} f\left(a_{j}, z\right)
$$

where $f(a, z)=\bar{a}(a-z) /|a|(1-\bar{a} z)$ converges uniformly in every closed set interior to the unit circle. It is well known that $\lim _{r \rightarrow 1} B\left(r e^{i \theta}\right)$ exists if $e^{i \theta}$ does not lie in an exceptional set $E$ of measure zero, and, in fact, that the limit is the same as $z$ approaches $e^{i \theta}$ from within any triangle with vertex at $e^{i \theta}$ lying within the closed unit disk. A discussion of the properties of Blaschke products may be found in [3].

O. Frostman $[2 ; 3]$ and L. Carleson [1] have considered Blaschke products for which

$$
\sum_{j \geq 1}\left(1-\left|a_{j}\right|\right)^{\alpha}<\infty,
$$

for some $\alpha<1$. They found that in this case the exceptional set could be further restricted. It was shown in [1] that for $e^{i \theta} \notin E$, an exceptional set of zero outer Hausdorff $\alpha$-dimensional measure, $\lim _{r \rightarrow 1} B\left(r e^{i \theta}\right)$ exists. Frostman [3] showed that for $\alpha<1 / 2$, the limit of the derivative of $B(z)$ exists and is finite except for a set whose capacity of order $2 \alpha$ is zero, ${ }^{2}$ if $z$ approaches $e^{i \theta}$ from within a triangle with vertex at $e^{i \theta}$ lying within the closed unit disk.

We show that, with the restriction (2), certain tangential approaches to the boundary may be allowed, provided that these boundary points lie outside of somewhat larger exceptional sets. The exceptional sets are described in terms of Hausdorff-Besicovitch dimension, which is defined as follows:

Let $C_{\mu}=\left\{0_{j}\right\}$ be a covering of $E$, where $\mu \geqq \max$ diam $0_{j}$. The outer Hausdorff $\gamma$-dimensional measure of $E$ is

Received by the editors July 16, 1959 and, in revised form, January 20, 1960 and May 27, 1960.

1 The work reported in this paper was performed by Lincoln Laboratory, a center for research operated by Massachusetts Institute of Technology with the joint support of the U. S. Army, Navy, and Air Force.

2 A set whose capacity of order $2 \alpha$ is zero is a set whose Hausdorff-Besicovitch dimension is at most $2 \alpha$, but not necessarily of zero outer Hausdorff $2 \alpha$-dimensional measure. The relations between these concepts are described in [1]. 


$$
L_{\gamma}(E)=\lim _{\mu \rightarrow 0} \underset{C_{\mu}}{\text { g.l.b. }} \sum_{j>0}\left(\operatorname{diam} 0_{j}\right)^{\gamma} .
$$

The Hausdorff-Besicovitch dimension of $E$, written $\operatorname{dim} E$, is defined as

$$
\operatorname{dim} E=\inf \left(\gamma \mid L_{\gamma}(E)=0\right)=\sup \left(\gamma \mid L_{\gamma}(E)=\infty\right) .
$$

We describe the tangential approaches to the boundary in terms of order of contact. A sequence $\left\{z_{j}\right\}$ is said to approach $e^{i \theta}$ with order of contact with the unit circle at most $\tau-1$ if $\lim _{j \rightarrow \infty} z_{j}=e^{i \theta}$ and $1-\left|z_{j}\right|>\left|\arg z_{j}-\theta\right|^{\tau}$. If $\lim _{i \rightarrow \infty} B\left(z_{i}\right)$ exists and is the same for all such sequences, we say that $\lim _{z \rightarrow e^{i \theta}} B(z)$ exists as $z$ approaches $e^{i \theta}$ from within the unit circle with order of contact with the unit circle at most $\tau-1$.

Our results are expressed in the

Theorem. We suppose $B(z)$ to be a Blaschke product defined by (1) with

$$
\sum_{j>0}\left(1-\left|a_{j}\right|\right)^{\alpha}<\infty .
$$

A. If $\alpha<1 / 2$, then for each $\tau$ with $1<\tau<(1-\alpha) / \alpha, \lim _{z \rightarrow e^{i \theta}} B(z)$ $=\lim _{r \rightarrow 1} B\left(r e^{i \theta}\right)$ as $z$ approaches $e^{i \theta}$ from within the unit circle with order of contact with the unit circle at most $(\tau-1)$, except possibly for a set $F_{\tau}$ on the unit circle with $\operatorname{dim} F_{\tau} \leqq \alpha \tau /(1-\alpha)$.

B. If $\alpha<1 / 3$, then for each $\sigma$ with $1<\sigma<(1-\alpha) / 2 \alpha, \lim _{z \rightarrow \theta^{i \theta}} B^{\prime}(z)$ $=\lim _{r \rightarrow 1} B^{\prime}\left(r e^{i \theta}\right)$ as $z$ approaches $e^{i \theta}$ from within the unit circle with order of contact with the unit circle at most $(\sigma-1)$, except possibly for a set $G_{\sigma}$ on the unit circle with $\operatorname{dim} G_{\sigma} \leqq 2 \alpha \sigma /(1-\alpha)$.

Proof of A. Let $b_{j}=1-\left|a_{j}\right|$. Then (2) becomes

$$
\sum_{j>0} b_{j}^{\alpha}<\infty \text {. }
$$

Choose $\beta$ with $\alpha<\beta<1-\alpha$. Let $\lambda_{j}$ be an arc on the unit circle with midpoint $a_{j} /\left|a_{j}\right|$ and length $2 b_{j}^{\beta}$. We define $E_{\beta}=\bigcap_{k \geq 1} U_{j \geq k} \lambda_{j}$. Since $\left\{\lambda_{j}\right\}_{j>k}$ is a covering of $E_{\beta}$ for each $k$,

$$
L_{\alpha / \beta}\left(E_{\beta}\right) \leqq \lim _{k \rightarrow \infty} \sum_{j>k}\left(\operatorname{diam} \lambda_{j}\right)^{\alpha / \beta} \leqq 2^{\alpha / \beta} \lim _{k \rightarrow \infty} \sum_{j>k} b_{j}^{\alpha} .
$$

Then by $\left(2^{\prime}\right)$ we have

$$
L_{\alpha / \beta}\left(E_{\beta}\right)=0 .
$$


Let $S_{j}$ be a circle with center at $a_{j}$, radius $b_{j}^{1-\alpha}$. Let $B_{n}(z)$ $=\prod_{j \leq n} f\left(a_{j}, z\right)$.

Lemma 1. For fixed $k, \lim _{n \rightarrow \infty} B_{n}(z)=B(z)$ uniformly for $z \notin \bigcup_{j>k} S_{j}$, $|z|<1$.

It will be sufficient to show that $\lim _{n \rightarrow \infty} \prod_{i>n} f\left(a_{i}, z\right)=1$ uniformly in the same region. Since $\lim _{j \rightarrow \infty} b_{j}=0$, it is easy to show that we can choose an $l$ so large that for $j>l, z \in S_{j}$, the following inequalities hold: $1 \geqq\left|f\left(a_{j}, z\right)\right|=\left|a_{j}-z\right| /\left|a_{j}\right|\left|z-\bar{a}_{j}^{-1}\right| \geqq\left|a_{j}-z\right| /\left(\left|a_{j}-\bar{a}_{j}^{-1}\right|\right.$ $\left.+\left|a_{j}-z\right|\right) \geqq 1-3 b_{j}^{\alpha} ;\left|\arg f\left(a_{j}, z\right)\right|=\left|\arg \left(a_{j}-z\right)-\arg \left(\bar{a}_{j}^{-1}-z\right)\right|<6 b^{\alpha}$. Then for $s>k, l, z \notin \bigcup_{j>s} S_{j}$, we have, $1 \geqq \prod_{j>s}\left|f\left(a_{j}, z\right)\right|$ $\geqq \prod_{j>8}\left(1-3 b_{j}^{\alpha}\right) \geqq 1-3 \sum_{j>s} b_{j}^{\alpha} \quad$ and $\left|\arg \prod_{j>s} f\left(a_{j}, z\right)\right|$ $\leqq \sum_{j>s}\left|\arg f\left(a_{j}, z\right)\right|<6 \sum_{j>s} b_{j}^{\alpha}$. By $\left(2^{\prime}\right)$, this establishes the lemma.

We define $P(s, \psi)$ to be the set where $1-|z|>|\arg z-\psi|^{s}$ and $\left|e^{i \psi}-z\right|<1$.

Lemma 2. If $e^{i \psi} \notin E_{\beta}$ and $s<(1-\alpha) / \beta, P(s, \psi)$ intersects at most a finite number of the $S_{j}$.

If $e^{i \psi} \notin E_{\beta}$, then there is a $k$ such that, for $j>k, e^{i \psi} \notin \lambda_{j}$. Hence it will be sufficient to show that, for sufficiently large $j, e^{i \psi} \oplus \lambda_{j}, z \in S_{j}$, we have $1-|z|<|\psi-\arg z|^{\text {s. Since }} \lim _{j \rightarrow \infty} b_{j}=0, \beta<1-\alpha$, and $s<(1-\alpha) / \beta$, we may choose $l$ so large that, for $j>l, b_{j}^{\beta}-2 b_{j}^{1-\alpha}>b_{j}^{\beta} / 2$ and $\left(b_{j}^{\beta} / 2\right)^{s}>2 b_{j}^{1-\alpha}$. Suppose $j>l$. If $z \in S_{j},\left|\arg a_{j}-\arg z\right|$ $\leqq \arcsin b_{j}^{1-\alpha} /\left|a_{j}\right| \leqq 2 b_{j}^{1-\alpha}$. Since $e^{i \psi} \notin \lambda_{j}, \quad\left|\arg a_{j}-\psi\right|>b_{j}^{\beta}$. Hence $|\psi-\arg z|^{\prime}>b_{j}^{\beta}-2 b_{j}^{1-\alpha}>b_{j}^{\beta} / 2$; so $|\psi-\arg z|^{\bullet}>\left(b_{j}^{\beta} / 2\right)^{\bullet}>2 b_{j}^{1-\alpha}$. However, for $z \in S_{j}, 1-|z| \leqq b_{j}+b_{j}^{1-\alpha}<2 b_{j}^{1-\alpha}$. Hence $|\arg z-\psi|^{\cdot}>1-|z|$.

We now suppose $e^{i \theta} \notin E_{\beta}, s<(1-\alpha) / \beta$. By Lemma 2, there exists a $k_{1}$ such that for $j>k_{1}$ and $z \in P(s, \theta), z \notin S_{j}$. By Lemma 1 , for any $\epsilon>0$, we can choose $k_{2}$ so large that, for $z \notin \bigcup_{j>k_{1}} S_{j}$ and $n>k_{2}$, $\left|B(z)-B_{n}(z)\right|<\epsilon$. Suppose $n>k_{2}$. Let $\left\{z_{j}\right\}$ be a sequence approachıng $e^{i \theta}$ as limit from within $P(s, \theta)$ with the restriction that the odd terms lie on the radius from the origin to $e^{i \theta}$. By Lemma 1 ,

$$
\limsup _{j \rightarrow \infty}\left|B\left(z_{j}\right)-B_{n}\left(z_{j}\right)\right| \leqq \epsilon .
$$

$B_{n}(z)$ is a rational function whose poles lie outside the unit circle; so it is continuous on the closed unit disk. Hence $\lim _{j \rightarrow \infty} B_{n}\left(z_{j}\right)$ exists. Since $\epsilon$ was chosen arbitrarily, $\lim _{j \rightarrow \infty} B\left(z_{j}\right)$ also exists and $\lim _{j \rightarrow \infty} B\left(z_{2 j}\right)$ $=\lim _{j \rightarrow \infty} B\left(z_{2 j-1}\right)$. This shows that for $s<(1-\alpha) / \beta, \lim _{z \rightarrow e^{i \theta}} B(z)$ $=\lim _{r \rightarrow 1} B\left(r e^{i \theta}\right)$ as $z$ approaches $e^{i \theta}$ from within $P(s, \theta)$ (and hence, by construction, with order of contact at most $(s-1)$ ), except possibly for the set $E_{\beta}$. 
We now fix $s=\tau$ and call our exceptional set $F_{\tau}$. For every $\beta^{*}$ $<(1-\alpha) / \tau, \lim _{z_{j} \rightarrow e^{i \theta}} B\left(z_{j}\right)=\lim _{r \rightarrow 1} B\left(r e^{i \theta}\right)$ for $\left\{z_{j}\right\} \subset P(\tau, \theta), e^{i \theta} \notin E_{\beta^{*}}$. Hence $F_{\tau} \subset E_{\beta^{*}}$. Using (3), and the fact that any covering of $E_{\beta^{*}}$ covers $F_{\tau}, L_{\alpha / \beta^{*}}\left(F_{\tau}\right) \leqq L_{\alpha / \beta^{*}}\left(E_{\beta^{*}}\right)=0$ for all $\beta^{*}<(1-\alpha) / \tau$. Hence $\operatorname{dim} F_{\tau} \leqq \alpha \tau /(1-\alpha)$.

ProOF OF B. We may write $B^{\prime}(z)=B(z) S(z)$ where $S(z)$ $=\sum_{j \geqq 1} a_{j}\left(1-\left|a_{j}\right|^{2}\right) /\left|a_{j}\right|^{2}\left(a_{j}-z\right)\left(z-\bar{a}_{j}^{-1}\right)$. Take $\gamma$ with $\alpha<\gamma$ $<(1-\alpha) / 2$. For each $j$ let $\sigma_{j}$ be an arc on the unit circle with midpoint $a_{j} /\left|a_{j}\right|$ and length $2 b_{j}^{\gamma}$, and define $D_{\gamma}=\bigcap_{k>0} \bigcup_{j \geq k} \sigma_{j}$. For each $j$ let $T_{j}$ be a circle with center at $a_{j}$ and radius $b_{j}^{(1-\alpha) / 2}$. Set

$$
S_{n}(z)=\sum_{j=1}^{n} a_{j}\left(1-\left|a_{j}\right|^{2}\right) /\left|a_{j}\right|^{2}\left(a_{j}-z\right)\left(z-\overline{a_{j}}\right) .
$$

The argument that led to (3) will show that

$$
L_{\alpha / \gamma}\left(D_{\gamma}\right)=0 \text {. }
$$

Lemma 3. For fixed $k, \lim _{n \rightarrow \infty} S_{n}(z)=S(z)$ uniformly for $z \notin \bigcup_{j \geqq k} T_{j}$, $|z|<1, z \notin\left\{a_{j}\right\}$.

Since $\lim _{j \rightarrow \infty} b_{j}=0$, it is not difficult to show that there is an $l$ such that, for $j \geqq l, z \notin T_{j}$,

$$
\begin{aligned}
\left.\left|a_{j}\left(1-\left|a_{j}\right|^{2}\right) /\right| a_{j}\right|^{2} & \left(a_{j}-z\right)\left(z-\bar{a}_{j}^{-1}\right) \mid \\
& \leqq 3 b_{j} /\left(\left|z-a_{j}\right|-\left|a_{j}-\bar{a}_{j}^{-1}\right|\right) b_{j}^{(1-\alpha) / 2} \leqq 6 b_{j}^{\alpha} .
\end{aligned}
$$

Then for $z \notin \bigcup_{j \geq l} T_{j},\left|S(z)-S_{l}(z)\right| \leqq 6 \sum_{j \geq l} b_{j}^{\alpha}$. The uniform convergence follows from $\left(2^{\prime}\right)$.

The same argument as that used to prove Lemma 2 will yield

Lemma 4. If $e^{i \psi} \notin D_{\gamma}$ and $s<(1-\alpha) / 2 \gamma, P(s, \psi)$ intersects at most a finite number of the $T_{j}$.

We take $e^{i \theta} \notin D_{\gamma}, s<(1-\alpha) / 2 \gamma$ and let $\left\{z_{j}\right\}_{j=1,2}, \ldots$ be a sequence approaching $e^{i \theta}$ from within $P(s, \theta)$ with odd terms on the radius from the origin to $e^{i \theta}$. Since $s<(1-\alpha) / 2 \gamma<(1-\alpha) / \gamma$, we may use the first theorem to see that $\lim _{j \rightarrow \infty} B\left(z_{j}\right)=\lim _{r \rightarrow 1} B\left(r e^{i \theta}\right)$. We use Lemmas 3 and 4 in the same way that Lemmas 1 and 2 were used above to show that $\lim _{j \rightarrow \infty} S\left(z_{j}\right)$ exists. From this we deduce that $\lim _{z \rightarrow \theta^{i \theta}} B^{\prime}(z)$ $=\lim _{r \rightarrow 1} B^{\prime}\left(r e^{i \theta}\right)$ as $z$ approaches $e^{i \theta}$ from within $P(s, \theta)$, except for the set $D_{\gamma}$.

We fix $\sigma$, and note that for $\gamma^{*}<(1-\alpha) / 2 \sigma$ and $e^{i \theta} \notin D_{\gamma^{*}}, \lim _{j \rightarrow \infty} B^{\prime}\left(z_{j}\right)$ $=\lim _{r \rightarrow 1} B^{\prime}\left(r e^{i \theta}\right)$ for $z_{j}$ approaching $e^{i \theta}$ from within $P(\sigma, \theta)$. Hence the exceptional set $G_{\sigma} \subset D_{\gamma^{*}}$. By (4) then, $L_{\alpha / \gamma^{*}}\left(G_{\sigma}\right) \leqq L_{\alpha / \gamma^{*}}\left(D_{\gamma^{*}}\right)=0$; so $\operatorname{dim} G_{\sigma} \leqq 2 \alpha \sigma /(1-\alpha)$. 


\section{BIBLIOGRAPHY}

1. L. Carleson, On a class of meromorphic functions and its associated exceptional sets, Thesis, University of Uppsala, 1950.

2. O. Frostman, Potentiel d'équilibre et capacité des ensembles avec quelques applications d la theorie des fonctions, Medd. Lunds Univ. Mat. Sem. vol. 3 (1935) pp. 1118.

3. - Sur les produits de Blaschke, Kungliga Fysiografiska Sällskapet i Lund förhandlingar vol. 12 (1942) pp. 169-182.

Lincoln Laboratory, Massachusetts Institute of Technology

\section{ON BOUNDED FUNCTIONS WITH ALMOST PERIODIC DIFFERENCES}

\section{RAOUF DOSS}

The aim of this paper is to generalize to groups the well-known Bohl-Bohr theorem, which states that if the indefinite integral, $F(x)$ $=\int_{a}^{x} f(t) d t$, of an almost periodic function $f(x)$, is bounded, then it is almost periodic (see [1] or [2]).

No expression of the form $\int_{0}^{x} f(t) d t$ is available in groups, but observing that $F(a+x)-F(x)=\int_{x}^{x+a} f(t) d t$ is easily proved to be almost periodic, whatever be the constant $a$, we are led to the following

THEOREM. Let $G$ be a multiplicative group and let the left differences $F(a x)-F(x)$ be right almost periodic for every $a \in G$, where $F$ is a given complex-valued function on $G$. If $F(x)$ is bounded then it is right almost periodic.

We recall that a real or complex function $\phi(x)$ is right almost periodic if, from every sequence $\left(c_{n}\right)$ we can extract a subsequence $\left(b_{n}\right)$ for which the functions $\phi\left(x b_{n}\right)$ converge uniformly in $G$. In that case to every $\epsilon>0$ there corresponds a finite number of elements of $G$, say $s_{1}, \cdots, s_{k}$, such that to every $t \in G$ we can associate an integer $i \leqq k$ for which

$$
\left|\phi(x t)-\phi\left(x s_{i}\right)\right|<\epsilon, \quad \text { whatever bel } x \in \mathbb{R} G .
$$

Proof of THE THEOREM. It is sufficient to consider the case of a real function. Suppose that $F(x)$ is not right almost periodic. Then there exists an $\alpha>0$ and a sequence $\left(c_{n}\right)$, such that, in every subse-

Received by the editors June 30, 1960. 